0038-1098(94)E0100-P

\title{
SURFACE PLASMON PEAK INTENSITY DEPENDENCE ON THE OXYGEN COVERAGE AT METAL SURFACES
}

\author{
A. Voskoboinikov* \\ Faculty of Applied Physics, University of Twente, P.O.Box 217, 7500 AE Enschede, The Netherlands \\ N. Nakhodkin, Yu. Kryn'ko, S. Kulik, P. Melnik, D. Sheka \\ Radiophysics Faculty, Kiev University, Vladimirskaya str.64, 252017 Kiev, Ukraine
}

(Received 11 October 1993 by D. Van Dyck)

\begin{abstract}
The dependence of the surface plasmon peak intensity on a submonolayer coverage of oxygen in the reflection electron energy loss spectra has been investigated for nonmonocrystalline aluminium, magnesium, and indium surfaces. It will be shown that the decrease of the surface plasmon peaks can be related to a modification of the surface plasmons dispersion relation. A simple model for this modification introduced by changes of the surface electron density profile is proposed. Parameters of this model will be determined from experimental data.
\end{abstract}

The surface plasmon (SP) peak shape and intensity in reflection electron energy loss (REEL) spectra depend on the near-surface electronic structure. Hence, REEL spectra contain important information about the near-surface electronic structure of solids. This is a reason, that the adsorption influence on the SP characteristics in the lowenergy region of the incident electrons $(<2000 \mathrm{eV})$ has been studied during the last three decades. ${ }^{1-7}$

The dependence of the SP peak shape and intensity on a submonolayer oxygen coverage $(\theta)$ for $\mathrm{Al}$ surface has been determined experimentally in pioneer works on REEL spectroscopy (see Ref. 1). It has been shown that for the initial stages of the oxygen adsorption (degree of coverage $\boldsymbol{\theta}<0.1$ monolayer (ML)), the intensity of the SP peak decreases rapidly, without visible changes of the peak position in the REEL spectrum. This effect also has been observed for other materials and under different conditions 3.6.7 although a systematic study of this phenomenon has not been performed yet

In the present work the SP peak intensity changes in REEL spectra for submonolayer oxygen coverages ( $\theta<$ $0.1)$ at non-monocrystalline surfaces of aluminium, magnesium and indium will be studied. We have found a close relation between SP peak changes and modifications of the SP dispersion relations. These modifications depend on the changes of the surface electronic density profile (SEDP). A simple model is proposed for the SEDP and its changes in the initial stage of oxygen deposition.

The experimental method for the receipt of angle resolved REEL spectra has been described in Ref. 8. For

\footnotetext{
* Permanent address: Radiophysics Faculty, Kiev University, Vladimirskaya str.64, 252017 Kiev, Ukraine
}

our purpose we have used an experimental geometry with fixed scattering angle $\theta=150^{\circ}$. The angles between the surface and the incident electron beam $(\gamma)$ and the angles of exit for the registered electrons $(\beta)$ satisfy equation $\beta=$ $180^{\circ}-\theta+\gamma$. The REEL spectra were measured for various magnitudes of the parameter $\varphi=\sin ^{-1} \gamma+\sin ^{-1} \beta(2.07<\varphi$ $<7.30$ ). The relative energy resolution was about $10^{-3}$ in the region of the incident electron energy $E=1000-1500$ $\mathrm{eV}$.

The working pressure in the chamber was about $10^{-10}$ Torr. Oxygen depositions at the metal surfaces have been performed at partial oxygen pressure of about $10^{-7}$ Torr. The oxygen input was realized by warming up a $\mathrm{CuO}$ source. The purity of the oxygen was checked by massspectrometry.

The REEL spectra, obtained at various oxygen coverages at the aluminium surface, are shown in Fig. 1. The quantity of adsorbed oxygen at the surfaces was determined by means of both work-function measurements (Anderson's method) and oxygen Auger spectroscopy measurements ${ }^{6}$ The observed changes in the workfunction and REEL spectra correspond to those described in other works. ${ }^{1-4,6,7}$

The obtained REEL spectra were assumed to consist of several contributions. The contributions are, with respect to their origin, divided into the following categories: elastically reflected electrons, electrons, which loose energy once or twice due to the excitation of surface and/or bulk plasmons, and electrons which had only suffered an energy loss through electron-electron interaction in the solid. The intensities of these peaks in the REEL spectrum were denoted $I_{e l}, I_{s}, I_{b}, I_{s b}, I_{s s}, I_{b b}, I_{e-e}$, respectively (see Fig. 1). Here we follow the procedure as described in detail in Ref.8. For all of the investigated 
spectra the relation $I_{S} / I_{e l}$ was determined with typical error bars of about $15 \%$.

The dependence of the SP-peak amplitude ratio $R=$ $A_{S} A_{O S}\left(A_{O S}\right.$ is the initial SP-peak amplitude for the clean metal surface) on the oxygen coverage for $\mathrm{Al}$ and $\mathrm{Mg}$ is shown in Fig. 2. This dependence is linear in $\theta$ for small $\theta$ values: $R(\theta) \approx 1-P \cdot \theta$. The SP peak position did not move visibly up to oxygen coverages of $\theta \sim 0.12$. The SS and SB peak intensity modifications follow the changes in the SP peak. Any change in the amplitudes of the other peaks was not observed in the above mentioned coverage range. For measured work-function $\varphi$ the linear dependence on $\theta\left(K(\theta)=\varphi / \varphi_{0} \approx 1-(\cdot \theta)\right.$ has been found for all three studied metals

The determined dependence of the SP peak intensity and work-function modifications for small oxygen coverages on $\mathrm{Al}$ and $\mathrm{Mg}$ are different from those observed on In. For the initial stage of oxygen adsorption at $\mathrm{Al}$ and $\mathrm{Mg}$ surfaces $R$ and $K$ decrease according to $P_{\mathrm{Al}}=4.2, C_{\mathrm{Al}}$ $=0.4$ and $P_{\mathrm{Mg}}=5.2, C_{\mathrm{Mg}}=2.5$, respectively. In the same time, the work-function of In increases $\left(C_{\text {In }}=-0.5\right)$, and $R$ changes are within the experimental error bars near $R \approx 1$ for $\theta \leq 0$.1. The largest magnitude of $R_{\operatorname{In}}$ corresponds to $R_{\text {In }} \approx 0.2$. Similar behaviour for $R$ and $K$ was found in Refs. $1,2,4,7$.

We shall explain our experimental data using the following assumptions. Firstly, SP peak shape as a function of electron energy loss, (in atomic units) $\Delta=\left(k^{2}-k^{2}\right) / 2$, is given by 9.10 :

$\mathrm{I}_{\mathrm{s}}(\Delta) \approx \mathrm{I}_{\mathrm{el}} \times \frac{k^{\prime}}{\pi^{2} k} \int d \Omega_{\mathbf{k}^{\prime}} \frac{q_{i !}}{\left[q_{! !}^{2}+q_{\perp}^{2}\right]^{2}} \operatorname{Im} g\left(q_{||}, \Delta\right)$

where $\boldsymbol{k}$ and $\boldsymbol{k}^{\prime}$ are the wave vectors of the incident and inelastically scattered electron, respectively; $d \Omega_{\mathbf{k}^{\prime}}$ is the solid angle of the electron scattering, $q_{\|}=k_{||}-k_{||}^{\prime}$ is the parallel and $q_{\perp}=k_{\perp}-k_{\perp}$ is the perpendicular component

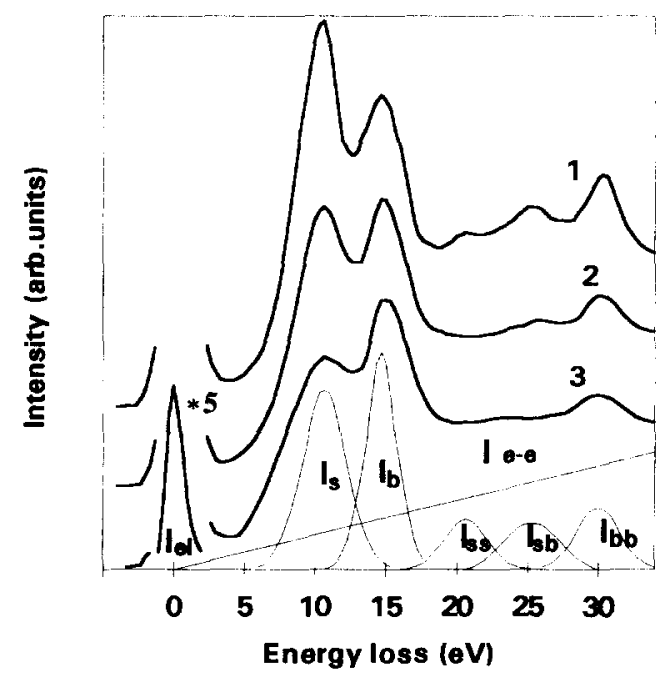

Figure 1. REEL spectra ( $\mathrm{E}=1300 \mathrm{eV}$ ) for $\mathrm{Al}$ with $\theta: 1-0 ; 2-0.07 ; 3-0.12$ of the surface momentum transfer, and $g\left(q_{\|}, \Delta\right)$ is the linear-response function of the seminfinite solid ${ }^{10}$ Secondly, in order to simplify the problem, in the $\Delta$-region near the SP peak maximum, we assume, that SP is a well defined quasiparticle, and

$\operatorname{Im} g\left(q_{1}, \Delta\right) \approx \pi \omega_{s}(q) \delta\left(\Delta-\omega_{s}\left(q_{\mid}\right)\right)$

where

$\omega_{s}(q) \approx\left(\omega_{p} / \sqrt{2}\right)(1+\alpha q)$

is the SP dispersion relation for long-wavelengths, $\omega_{p}$ - the bulk plasma frequency. Using these assumptions the $I_{S}(\Delta)$ dependence on the conditions at the surface appears in the coefficient $\alpha$, since it is a functional of the SEDP

$\alpha=-\left[d_{\perp}\left(\omega_{p} \sqrt{2}\right)-d\right] / 2$

where $d_{\perp}(\omega)$ is the centroid of the induced surface charge and $d_{\|}$is the gradient of the metal's unperturbed SEDP as determined by Feibelman. ${ }^{11.12}$ We can obtain using Eqs. (1) $-(3)$ that the maximum of the $I_{s}(\Delta)$ (for electrons at normal incidence) is located at

$\Delta_{\max } \approx\left(\omega_{p} / \sqrt{2}\right)\left(1+\alpha \omega_{p} / \sqrt{2} k\right)$

and $I_{S}\left(\Delta_{\max }\right)$ depends on $\alpha$ according to

$A_{s}=\mathrm{I}_{\mathrm{s}}\left(\Delta_{\max }\right) \approx$ const $\times \alpha^{-1}$

For the initial stages of adsorption, we can represent function $\alpha(\Theta)$ as follows

$\alpha \approx \alpha_{0}(1+P \theta)$

where the sign " 0 " refers to the clean surface and $P$ is a functional of the SEDP. In this case

$A_{s} / A_{\mathrm{so}} \approx \mathrm{l}-P \theta$

and

$\Delta_{\max } / \Delta_{\max 0} \approx 1+S \theta$

where

$S=P \alpha_{0} \omega_{p} / \sqrt{2} k\left(1+\alpha_{0} \omega_{p} \sqrt{2} k\right)$

From Eq.(10) it follows that the dependence $\Delta_{\max }(\theta)$

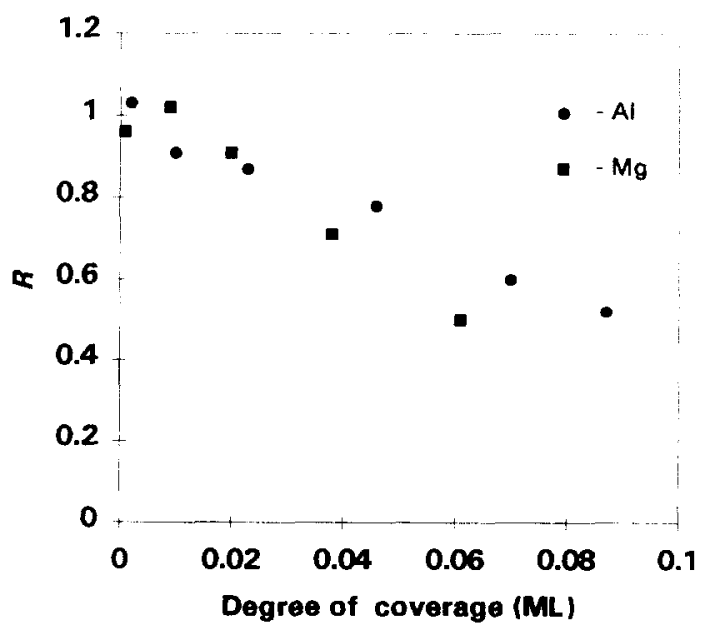

Figure 2. Measured $R(\theta)$ (exposure: for $\mathrm{Al}$ $\mathrm{L}=200 \times \theta$, for $\mathrm{Mg}-\mathrm{L}=62 \times \theta)$ 
Table

\begin{tabular}{|l|l|l|l|l|l|l|l|l|l|}
\hline Metal & $P$ & $c$ & $\alpha_{0}(\mathrm{a} u)$ & $D_{0}$ (a.u.) & $x_{0}$ & $d_{0}\left(\mathrm{~A}^{0}\right)$ & $d_{\mathbf{a}}\left(\mathrm{A}^{0}\right)$ & $\mathrm{a}$ & Q (a.u.) \\
\hline $\mathrm{Al}$ & 4.2 & 0.4 & $-0.7^{\mathrm{a}}$ & $0.22^{\mathrm{b}}$ & 0.83 & 1.64 & 0.63 & -0.66 & 0.32 \\
\hline $\mathrm{Mg}$ & 5.2 & 2.5 & $-1.1^{\mathrm{a}}$ & $0.11^{\mathrm{b}}$ & 0.74 & 1.47 & 0.8 & -1.05 & 0.56 \\
\hline $\mathrm{In}$ & $0.2^{*}$ & -0.5 & $-0.8^{\mathrm{a}}$ & $0.17^{\mathrm{b}}$ & 0.79 & 1.6 & -1.8 & -0.36 & 0.02 \\
\hline
\end{tabular}

*Max. $P$ for In

aReference 14

bReference 13

should to be much more weaker than $A_{s}(\theta)$. For $\mathrm{Al}$ and at an incident electron energy of $1300 \mathrm{eV}$, we find $S / P \approx \alpha_{0} \times$ 0.04 .

We have determined that the changes of the SP peak amplitude are related to a modification of the SEDP through a modification of the SP dispersion relation. It is attractive to reconstruct characteristics of the SEDP using experimental data and the simplest available theoretical model. For this purpose we use the "jellium model" with a $\delta$-like oxygen coverage. The localization of a part of the electronic charge is a suitable way to describe the adsorption of more electronegative atoms on a metallic surface ${ }^{13}$ In this model the electronic density $n_{-}(z)$ and the smeared positive charge of the ionic cores of the solid n. (z) (located at $z>0$ ) are

$n_{-}(z)=n_{s} \theta\left(z+d_{0}-z_{d}\right)+$

$+\left(n_{0}-n_{s}\right) \Theta\left(z-z_{d}\right)+N_{\mathrm{a}} \delta\left(z+d_{\mathrm{a}}\right)$,

$n_{+}(z)=n_{0} \theta(z)$

where $n_{s}$ and $n_{0}$ are uniform densities in a near-surface region and in the bulk, respectively; $d_{0}$ is an effective thickness of the near-surface region with electronic density $n_{s} ; z_{d}$ is an effective thickness of the near-surface electron depletion region in the solid; $N_{\mathrm{a}}$ and $d_{\mathrm{a}}$ are an effective surface density of the adsorbate charge and centroid of this charge, respectively, and $\Theta(z)$ is the Heaviside stepfunction. Finally, we assume that $N_{\mathrm{a}} \approx \mathrm{Q} \cdot \theta /\left(n_{0} \cdot \mathrm{s}_{0}\right)$ and $n_{s} / n_{0}=x(\theta)=x_{0}(1+\mathrm{a} \cdot \theta)$, where $Q$ is an effective charge of the adsorbed ions; $s_{0}$ is an average one-atom metal area at the surface. Eigenfrequencies of the adsorbed ions are much smaller than $\omega_{p}$, and $\mathrm{Q}$ may be introduced as a nonoscillating charge. For the represented model we can determine $\alpha_{0}$ and $P$ as functions of the parameters of the model

$\alpha_{0}=d_{0} x_{0}\left(x_{0}-1\right) /\left(2 x_{0}-1\right)$

$P=\mathrm{a}\left(1-2 x_{0}+2 x_{0}^{2}\right) /\left(x_{0}-1\right)\left(2 x_{0}-1\right)$.

Since the experimental information on the workfunction should be described by the model too, we now calculate the dipole part of the work-function as a function of the $\theta^{13}$ If we take into account the system neutrality condition, we obtain

$D(\boldsymbol{\theta})=4 \pi \int d z z\left[n_{+}(z)-n_{-}(z)\right]=D_{0}(1-C \theta)$.

where $D_{0}=2 \pi n_{0} d_{0}{ }^{2} x_{0}\left(1-x_{0}\right)$ and $\left(=\mathrm{a}\left(1-2 x_{0}+2 d_{\mathrm{a}} / d_{0}\right) /\left(x_{0}-1\right)\right.$

In order to determine the parameters $x_{0}, d_{0}, a_{0}$ and $d_{\mathrm{a}}$ we can use both experimental values for $P$ and $C$, and the known literature data for $\alpha_{0}$ and $D_{0}$. The results of this calculation are shown in the Table. It is quite remarkable that the magnitudes of the obtained parameters are in a very reasonable region.

The near-surface effective electronic charge decreases for all materials when the total adsorbate electronic charge is increased. However, there is a difference between $\mathrm{Al}$ and $\mathrm{Mg}$, on one hand, and In on the other hand, with respect to the determined position of the oxygen atom at the surface. The oxygen atom is located outside the positive core for $\mathrm{Al}$ and $\mathrm{Mg}$ surfaces, whereas for the In surface $d_{\mathrm{a}}$ corresponds to a configuration, where the $\mathrm{O}$-atom is located inside the near-surface region of the In positive ionic core charge $(z>0)$. This explains the sign of the $C_{\text {In }}$ and is in good agreement with other experimental data published by other authors. ${ }^{7}$

The proposed model is too simple for far-reaching conclusions, but it shows a possibility to obtain additional information from the REEL data. It will therefore be very interesting to measure the $\alpha(\theta)$ dependence at the initial stages of oxygen adsorption and to compare it with Eq.(7)

Summarizing, we can to conclude that changes of the SP peak intensity in the REEL spectra for the initial stages of oxygen adsorption are related to a modification of the SP dispersion relation. This modification corresponds to a change of the SEDP parameters and there is a possibility to determine the SEDP parameters using the experimental REEL spectroscopy data. The expression for the SP dispersion relation and the simple jellium model, described above, yields reasonable magnitudes for the SEDP and oxygen-adsorbate parameters in the case of the nonmonocrystalline surfaces $\mathrm{Al}, \mathrm{Mg}$ and In

Acknowledgement - This work was supported by the Netherlands Organisation of Pure and Scientific Research (NWO). Dr. H.J.W. Zandvliet, Dr. C.M.J. Wijers, and Dr. E. G. Keim comments are greatly appreciated. 


\section{References}

1. C.J. Powell and J.B. Swan, Phys.Rev. 118 (1960) 640.

2. C. Bendorf, G. Keller, H. Seidel and T.Thieme, Surf.Sci. 67 (1977) 589.

3. H. Namba, J. Darville and J.M. Gilles, Solid State Commun. 34 (1980) 287.

4. A. Hoffman, T. Mavin and M. Folman, Surf.Sci. 193 (1988) 513.

5. H. Ishida and A. Liebsch, Phys.Rev. B 45 (1992) 6171

6. N.G. Nakhodkin, A.M. Voskoboinikov, Yu.N Kryn'ko, S.P. Kulik, P.V. Melnik and D.I.Sheka, Fiz. Tverdogo Tela (Russian) 33 (1992) 3359.

7. Dai-Xuan Dai and Fu-Rong Zhu, Appl.Surf.Sci. 59 (1992) 195
8. Yu. Kryn'ko, P.V. Melnik and N.G. Nakhodkin, Izv AN SSSR (Russian), ser.Fiz., 33 (1969) 481.

9. H. Ibach and D.L. Mills, Electron-Energy Loss Spectroscopy and Surface Vibration (Academic, San Francisco, 1982).

10. B.N.J. Person and E.Zaremba, Phys.Rev. B 31 (1985) 1863.

11. P.J. Feibelman, Prog.Surf.Sci. 12 (1982) 287.

12. P.J. Feibelman and K.D. Tsuei, Phys.Rev. B 41 (1990) 8519.

13. N.D. Lang, in: Theory of the inhomogeneous electron gas, Eds. by S. Lundquist and N.M. Mach (Plenum Press, New York, 1983) p.323.

14. A.Liebsch, Phys.Rev. B 36 (1987) 7378 